\title{
Gamificación en la asignatura Derecho Romano: un estudio de caso Gamification in a Roman Law course: a case study
}

\author{
Raquel Escutia Romero ${ }^{1}$, Sonia Pamplona Roche ${ }^{2}$ \\ raquel.escutia@uam.es,sonia.pamplona@udima.es \\ ${ }^{1}$ Departamento de Derecho Privado, Social \\ y Económico \\ Universidad Autónoma de Madrid (UAM) \\ Madrid, España \\ ${ }^{2}$ Departamento de Ingeniería Informática \\ y Tecnología Educativa \\ Universidad a Distancia de Madrid, UDIMA \\ Madrid, España
}

\begin{abstract}
Resumen- Este trabajo describe dos experiencias de gamificación en la asignatura Derecho Romano: una síncrona, realizada con la herramienta Kahoot y otra asíncrona, llevada a cabo con la herramienta Lección del sistema de gestión del aprendizaje Moodle. El número de estudiantes matriculados en dicha asignatura es de 236. El objetivo del estudio es analizar los efectos que han tenido las técnicas de gamificación en el proceso de aprendizaje de los estudiantes. La metodología de investigación es un estudio de caso, en el que se han utilizado los siguientes instrumentos de recogida de datos: observación participante, un cuestionario y 9 pruebas de evaluación. Los resultados muestran tres efectos principales: diversión, motivación y mejora del aprendizaje, que actúan de forma desigual entre los estudiantes.
\end{abstract}

\section{Palabras clave: gamificación, Derecho Romano, evaluación formativa}

Abstract- This work describes two gamification experiences in a Roman Law course: a synchronous one, implemented with the Kahoot tool and an asynchronous one, carried out with the lesson tool of the Moodle learning management system. The number of students enrolled in this subject is 236 . The study aims to analyze the effects that gamification techniques have had on the learning process of students. The research methodology is a case study and the following data collection instruments have been used: participant observation, a questionnaire and 9 tests. The results show three main effects: fun, motivation and improved learning, which act unequally among students.

\section{Keywords: gamification, Roman Law, formative assessment}

\section{INTRODUCCIÓN}

La aplicación de las técnicas de gamificación en el área de educación ha mostrado resultados positivos (Dicheva, Dichev, Agre \& Angelova, 2015). Dichas técnicas consisten en el uso de elementos para el diseño de juegos en entornos que no son juegos (Deterding, 2015). Uno de los principales beneficios del uso de la gamificación en el contexto educativo es la motivación del estudiante en su proceso de aprendizaje (Bodnar, Anastasio, Enszer \& Burkey, 2016).

Por otra parte, existen evidencias de que la realización de pruebas de evaluación mejora el aprendizaje (Adesope, Trevisan \& Sundararajan, 2017).

Este trabajo tiene como objetivo combinar los beneficios de las dos técnicas mencionadas: la gamificación y la realización de pruebas de evaluación. Para ello, se han diseñado dos tipos de pruebas de evaluación gamificadas. El objetivo es motivar al estudiante para la realización de las pruebas y, por tanto, mejorar su aprendizaje a través de las mismas.

El primer tipo de prueba es una prueba síncrona, que se realiza en el contexto de una clase presencial para comprobar la comprensión de los contenidos expuestos. Este tipo de prueba se ha llevado a cabo en los últimos minutos de las clases magistrales durante 8 sesiones y se ha llevado a cabo con la herramienta Kahoot.

El segundo tipo de prueba es una prueba asíncrona, que los estudiantes han realizado a través del sistema de gestión de aprendizaje moodle como repaso de la asignatura y durante el periodo de preparación del examen final presencial.

Ambas pruebas tienen un propósito formativo, es decir, se han usado con la intención de mejorar el aprendizaje, lo que contrasta con las pruebas sumativas, que tienen como finalidad calificar y certificar al final de un periodo formativo (Bloom, Madaus \& Hastings, 1981).

Una característica de las publicaciones de gamificación en el área de educación es la escasez de estudios empíricos o la falta de rigor de los mismos (Dicheva et al., 2015). Muchos trabajos tienen como único objetivo la descripción de mecanismos y dinámicas de juego y como consecuencia, se desconoce la efectividad de las técnicas descritas.

En cuanto al área de conocimiento en la que se han desarrollado los trabajos de gamificación, la mayoría se concentra en las áreas de Ciencia y Tecnología (programación de juegos, matemáticas, informática, tecnología e ingeniería) (Dicheva et al., 2015). En este trabajo presentamos un estudio empírico en un área muy distinta: el área de las Ciencias Jurídicas y, en concreto, en la asignatura de Derecho Romano.

Los estudios acerca de las técnicas de gamificación en el área de Derecho son escasos y generalmente no son estudios empíricos. Un ejemplo es un trabajo acerca de la realización de una aplicación basada en un juego para la Escuela de Derecho de la Universidad de Westminster de Londres, que se limita a la descripción de la aplicación y no incluye recogida ni análisis de datos para avalar la efectividad de la experiencia (Bouki, Economou \& Kathrani, 2014).

En resumen, la contribución de este trabajo es presentar un estudio empírico en el que se analizan los efectos en el proceso de aprendizaje de la aplicación de las técnicas de 
gamificación en la asignatura de Derecho Romano. Hasta donde llega nuestro conocimiento, no existen trabajos acerca de la gamificación de esta asignatura. La puesta en marcha de la experiencia y la metodología utilizada pueden ser tomadas como referencia para otros profesores del área interesados en gamificar sus asignaturas.

\section{CONTEXTO}

El presente trabajo muestra la puesta en marcha y desarrollo de dos experiencias de innovación docente dentro del ámbito de las Ciencias Jurídicas, en concreto, en la asignatura de Derecho Romano, asignatura de Formación Básica de 6 créditos ECTS, impartida en el primer semestre del primer curso del Grado de Derecho de la Universidad Autónoma de Madrid (UAM) en un entorno presencial. Dichas experiencias se han realizado en el curso académico 2016/2017 con un total de 236 estudiantes matriculados. El perfil del estudiante es el de joven de entre 17-18 años, recién accedido a la Universidad con una nota de corte media de 7,807 y con una distribución por género de 151 mujeres (63,98\%) y 85 varones (36,01\%).

Conforme a la Ordenación académica propia de la Facultad de Derecho de la UAM las clases se dividen en Magistrales de carácter, en general, más teórico y Seminarios con un contenido más práctico de aplicación de lo tratado en las Magistrales. Las clases Magistrales se impartieron cada miércoles durante 14 semanas, en dos sesiones desde las $8.30 \mathrm{~h}$ a $10.30 \mathrm{~h}$ (con $10 \mathrm{~min}$. de descanso entre ambas sesiones). Esta división se proyecta en la Evaluación de la Asignatura que distingue ponderando un 30\% la Evaluación Continua efectuada sobre los Seminarios y un 70\% la Prueba Final sobre todo el contenido práctico y teórico de la Asignatura, fundamentalmente de lo impartido en las Magistrales.

Para promover la atención en las clases Magistrales y la motivación para el aprendizaje de la asignatura se han implementado dos experiencias didácticas innovadoras: la utilización de Kahoot en las clases Magistrales y la creación de un Juego a través de una Lección en Moodle para el repaso final de la materia objeto de evaluación del Examen final que supone un $70 \%$ de la nota final de la asignatura.

La pregunta de investigación que ha guiado este estudio es la siguiente: ¿Qué efectos han tenido las experiencias de aprendizaje diseñadas sobre el proceso de aprendizaje de los estudiantes?

\section{DESCRIPCIÓN}

En este apartado se describen cómo se ha realizado la gamificación de cada una de las pruebas de evaluación, los elementos de los juegos incluidos en cada experiencia, y la metodología de investigación utilizada.

\section{A. Gamificación de la prueba de evaluación síncrona: herramienta Kahoot}

Para diseñar la gamificación de las sesiones síncronas se utilizó la herramienta Kahoot que permite convertir una prueba de evaluación en un juego de competición entre los estudiantes. El profesor proyecta una serie de preguntas en una pantalla con la ayuda de un ordenador o tableta y los estudiantes deben responder a ellas de la forma más rápida y correcta posible en un tiempo limitado. Para responder a las preguntas, los estudiantes necesitan usar un dispositivo móvil (teléfono inteligente, tableta $\mathrm{u}$ ordenador portátil). Cuando finaliza el tiempo destinado a cada pregunta se visualiza un gráfico con la distribución de las respuestas de los estudiantes que permite al profesor comprobar la comprensión de cada uno de los conceptos incluidos en las preguntas. Además, se muestran los estudiantes que han obtenido mejor puntuación en cada pregunta. Los estudiantes compiten entre sí y al final del juego se anuncia el ganador que será quién haya respondido más preguntas correctas usando la menor cantidad de tiempo. En la figura 1 puede verse un ejemplo de una de las preguntas de la prueba de evaluación realizada con esta herramienta.

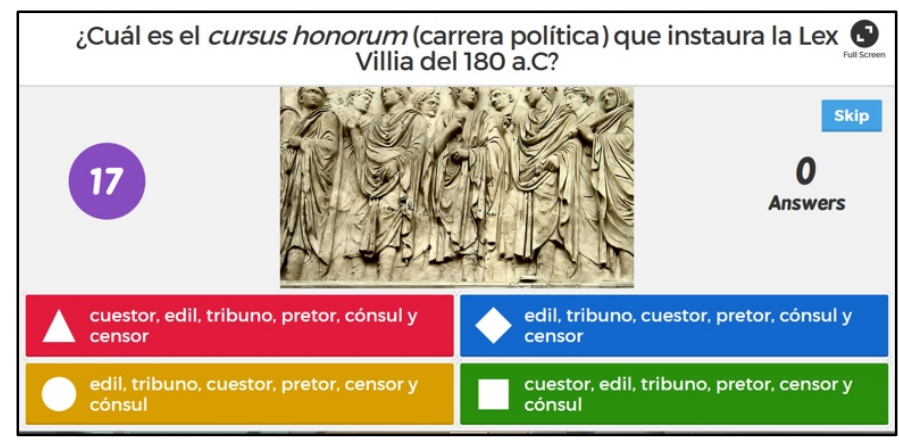

Figura 1. Pregunta de la evaluación realizada con Kahoot

\section{B. Gamificación de la prueba de evaluación asíncrona: herramienta Lección de Moodle.}

La gamificación de la prueba de evaluación asíncrona que serviría como repaso se llevó a cabo mediante el diseño un juego que fue implementado con la herramienta Lección del sistema de gestión del aprendizaje moodle. El juego fue denominado "El Templo de la Jurisprudencia” en alusión al tablero creado tomando como punto de partida una fotografía de la cúpula del Panteón de Agripa (figura 2).

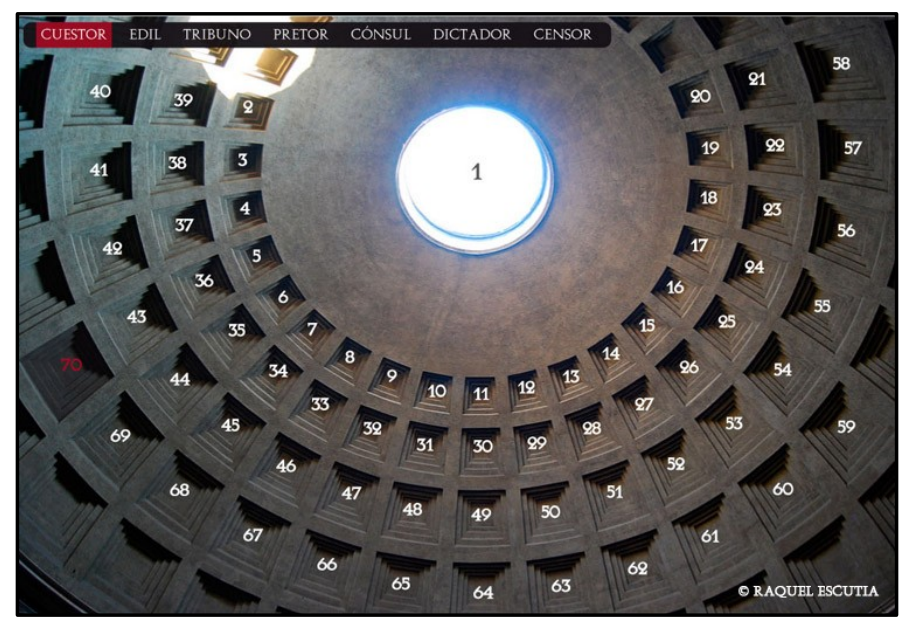

Figura 2. Pantalla de "El Templo de la Jurisprudencia”

El contenido del juego está estructurado en 70 casillas agrupadas en los 7 bloques temáticos que constituyen la asignatura de Derecho Romano (1. Derecho Público, 2. Derecho de la Persona, 3. Negocio Jurídico, 4. Derecho Procesal, 5. Derechos Reales, 6. Derechos de Obligaciones y Contratos y 7. Derecho de Familia y Sucesiones).

Los temas están enlazados conformando una historia ficticia ambientada en la época romana que el estudiante sigue en primera persona, creando así una narrativa con la intención de atraer y mantener la atención del estudiante. En cada casilla se 
tiene la oportunidad de sumar puntos si se contesta de forma correcta a una pregunta con múltiple opción y se tienen dos intentos por cada casilla. Cada uno de los bloques en los que está dividido el juego, está asociado con un rango en la carrera política romana. De este modo, al ir avanzando en las preguntas de cada bloque los estudiantes adquieren una nueva y más alta magistratura: cuestor, edil, tribuno, pretor, cónsul y censor (figura 2). Estos rangos suponen una recompensa que puede motivar a los estudiantes a continuar el juego.

\section{Elementos de los juegos}

A continuación se realiza un análisis de los elementos de los juegos (Bodnar et al., 2016) que están presentes en cada una de las experiencias de gamificación diseñadas (tabla 1).

Tabla 1. Elementos de los juegos presentes en las experiencias de gamificación llevadas a cabo.

\begin{tabular}{|l|l|l|}
\hline Elementos de los juegos & Kahoot & $\begin{array}{l}\text { Lección } \\
\text { moodle }\end{array}$ \\
\hline Retroalimentación inmediata & Sí & Sí \\
\hline Participación voluntaria & Sí & Sí \\
\hline Competición & Sí & No \\
\hline Cooperación & Sí & No \\
\hline Narrativa & No & Sí \\
\hline Recompensas & Sí & Sí \\
\hline & & \\
\hline
\end{tabular}

En ambas experiencias de gamificación existe retroalimentación inmediata, puesto que el estudiante conoce al instante si ha contestado de forma correcta a la pregunta o no.

La participación en ambos juegos ha sido voluntaria, dado que hacer un juego obligatorio puede ocasionar que la experiencia no sea agradable ni divertida (Salen \& Zimmerman, 2003).

Los conceptos de cooperación y competición sólo existen en las pruebas de evaluación síncrona realizadas con Kahoot puesto que está previsto que la Lección de repaso se realice de forma individual.

La narrativa es un elemento importante porque puede atraer y mantener la participación de los estudiantes. En nuestro caso, este elemento está presente en la Lección en la que los temas están enlazados mediante una historia ficticia, pero no en los Kahoot, que constan de preguntas independientes.

Por último, las recompensas existen tanto en los Kahoot como en la Lección. En los primeros la recompensa sería el sentimiento de victoria que se puede producir al contestar de forma correcta a cada pregunta o al resultar ganador entre toda la clase. En la Lección, las recompensas son el sentimiento de logro que los estudiantes experimentan al completar cada uno de los siete niveles del juego en los que alcanzan rangos de la carrera política romana cada vez más elevados y el Triunfo.

\section{Metodología de investigación}

La metodología de investigación de este trabajo es el estudio de caso, que se define como la investigación intensiva de un único objeto de indagación social, tal y como un aula (Stake, 1978). La ventaja principal de un estudio de caso es que el investigador puede descubrir hechos o procesos que probablemente pasaría por alto si utilizara otros métodos de investigación más superficiales (Wittrock, 1989). Una de las características principales de un estudio de caso es que se combinan diferentes técnicas y se triangulan sus resultados con el propósito de iluminar un caso desde diferentes ángulos.

A continuación se citan los instrumentos de recogida de datos utilizados en el estudio:

- Observación participante. Se ha llevado a cabo en la experiencia innovadora con Kahoot y fue realizada por la profesora de la asignatura quién registró la información relacionada con las conductas observadas en cada una de las sesiones. Las unidades de observación fueron los estudiantes presentes en el aula en el momento de la puesta en marcha del Kahoot. Las conductas a observar en los estudiantes fueron: niveles de interés y participación y comportamiento.

- Pruebas de evaluación. En la experiencia de Kahoot se utilizaron 8 pruebas de evaluación con 6 preguntas cada una sobre cada uno de los temas cubiertos en las clases magistrales (proceso, cosas, posesión y derechos reales, derecho de propiedad, derechos reales, obligaciones y contratos, contratos reales y contratos consensuales). En la Lección se utilizó una única prueba de evaluación en la que se incluyeron preguntas de todos los bloques temáticos de la asignatura Derecho Romano.

- Cuestionario de satisfacción. El cuestionario de satisfacción que se puede ver como anexo, valora la satisfacción de las dos experiencias de innovación docente y contiene tanto preguntas cerradas (para analizar los efectos de la gamificación ya conocidos) como abiertas (para descubrir nuevos efectos). Las preguntas cerradas usan una escala Likert de 5 categorías (Completamente de acuerdo, más bien de acuerdo, neutral, más bien en desacuerdo, completamente en desacuerdo).

\section{E. Análisis de datos}

Las preguntas del cuestionario que se han respondido mediante una escala Likert se han analizado de forma cuantitativa con la ayuda de la herramienta Microsoft Excel, y las preguntas abiertas y los registros de la observación participante se han analizado con la ayuda del software de análisis de datos cualitativo ATLAS.ti. El proceso de análisis de datos cualitativos se ha realizado en dos fases. En primer lugar, se ha llevado a cabo una codificación abierta etiquetando cada uno de los fragmentos de texto con un código creado de acuerdo al contenido del texto. Por último, se han agrupado los códigos obtenidos en la fase anterior para dar respuesta a la pregunta de investigación planteada.

\section{Resultados}

En este apartado se describen los datos de participación en las dos experiencias de gamificación y los resultados preliminares del estudio correspondientes a la observación participante y a la encuesta de satisfacción. En un trabajo posterior se darán a conocer los resultados del análisis de las pruebas de evaluación llevadas a cabo por los estudiantes.

\section{A. Participación en las experiencias de gamificación}

El número total de estudiantes matriculados en la asignatura fue de 236. Las 6 pruebas de evaluación implementadas 
mediante la herramienta Kahoot fueron llevadas a cabo por 94, 86, 80, 60, 53, 43, 41 y 48 estudiantes respectivamente. Las pruebas fueron realizadas por aproximadamente el $80 \%$ de los estudiantes que asistieron a clase.

La Lección de Moodle fue realizada por 158 estudiantes, de los cuales 94 llegaron al final del juego. Esto supone una participación del $67 \%$ en esta actividad voluntaria.

\section{B. Resultados de la observación participante}

A partir de la sexta semana del curso, en la Magistral número 6, con los estudiantes ya plenamente incorporados e integrados en la Facultad, se planteó por sorpresa al final de la clase la realización de un Kahoot. Previamente se había preguntado si tenían dispositivos móviles (smartphones, tablets $\mathrm{y} / \mathrm{u}$ ordenadores). Se les indicó cómo descargar la aplicación en el móvil y cómo acceder desde el ordenador.

El primer Kahoot se planteó como una prueba y como consecuencia algunos estudiantes no se registraron con sus nombres reales, sino con apodos $\mathrm{y}$ distintas bromas (“Justiniano”, "Elnacho”, “El bicho”, “Tu amante”). El uso de apodos de mantuvo a lo largo de todos los Kahoot, aproximadamente un $10 \%$ de los estudiantes no usó su nombre real. Este comportamiento se podría explicar por el deseo de anonimato y/o por el ambiente de diversión generado por los Kahoots. Para mejorar este aspecto se propone para futuras ocasiones que la actividad siga siendo voluntaria, pero pueda elevar unas décimas la calificación final de la asignatura.

La experiencia captó inmediatamente la atención de los estudiantes y causó furor desde las primeras respuestas, que conforme se iban resolviendo y verificando provocaban más entusiasmo y griterío en los estudiantes. Una vez terminado el Kahoot se identificaba al ganador/a y se le felicitaba y daba un aplauso por parte de la clase junto con los otros dos finalistas según el ranking. Ello provocaba satisfacción y en algunos casos vergüenza en los estudiantes más introvertidos. El furor y entusiasmo primero se fue apaciguando a lo largo de las sesiones, pero se mantuvo el griterío cada vez que se daba a conocer la respuesta correcta a cada una de las preguntas.

Se observó también que había estudiantes que se agruparon con otro estudiante porque no disponían de teléfono móvil. Y hubo también estudiantes que aún teniéndolo no participaron en los Kahoot, quizá por no buscar y descargar la aplicación.

El seguimiento de la experiencia fue bastante continuado e incluso hubo estudiantes que solicitaban al final de la clase la realización del Kahoot. De hecho, una estudiante quedó encargada de avisar del tiempo restante en clase para que siempre pudiera realizarse. El Kahoot se realizó en los cinco últimos minutos de cada clase magistral.

\section{Resultados del cuestionario de satisfacción}

A continuación se presentan los resultados del cuestionario de satisfacción. Se analizan de forma separada las preguntas con escala Likert, las preguntas sobre Kahoot y las preguntas acerca de la Lección Moodle. Este cuestionario fue respondido por 55 estudiantes, un $23 \%$ del total de estudiantes matriculados en la asignatura y aproximadamente un $50 \%$ de los estudiantes que han participado en ambas experiencias de gamificación.

\section{Preguntas escala Likert}

Las preguntas que han alcanzado mayor acuerdo entre los estudiantes han sido las relacionadas con la diversión.

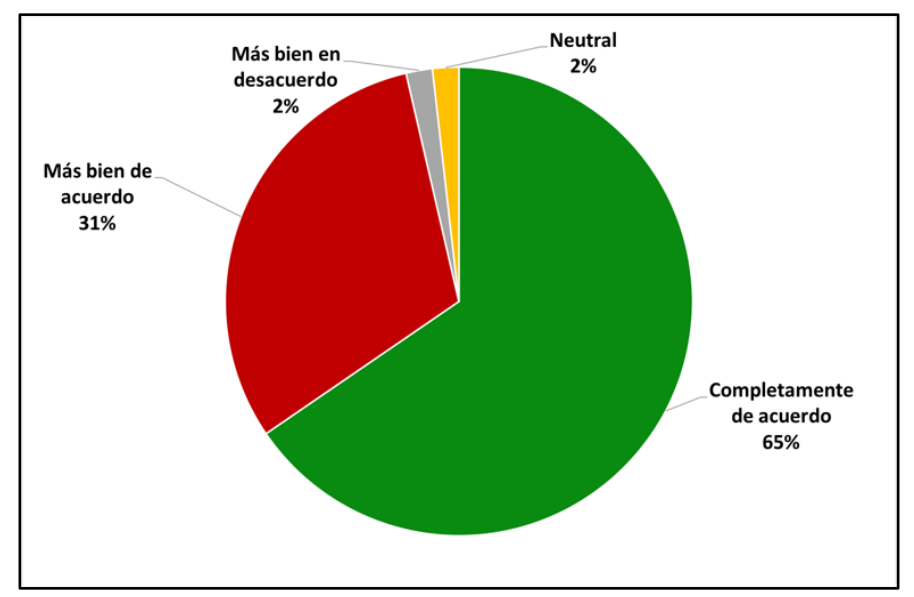

Figura 3. "Ha sido divertido competir con otros”.

La figura 3 muestra los resultados de la pregunta "Ha sido divertido competir con otros”. Sólo hay un estudiante (2\%) que ha respondido que está más bien en desacuerdo y otro (2\%) se muestra neutral. 36 estudiantes (65\%) están completamente de acuerdo con la afirmación y, por tanto, les divierte la competición y 17 estudiantes (31\%) están más bien de acuerdo. Esto últimos pueden tener alguna reserva en cuanto a las actividades competitivas.

Si se comparan estos resultados con los de la pregunta "Ha sido divertido jugar todos juntos en el aula” se comprueba que 43 estudiantes (78,2\%) están completamente de acuerdo, 8 estudiantes están más bien de acuerdo (14,5\%) y 4 estudiantes $(7,3 \%)$ se muestran neutrales. En consecuencia, parece que este grupo de estudiantes se muestra más a favor de la cooperación que de la competencia.

En cuanto a la motivación, 21 estudiantes (38,4\%) están completamente de acuerdo en que Kahoot les ha motivado tanto a asistir a clase como a permanecer más atentos durante las clases. Con respecto al resto de categorías de la escala Likert, los resultados muestran que la experiencia ha motivado más a los estudiantes a prestar más atención en clase que a asistir a las clases. En concreto, en la pregunta "El uso de Kahoot me ha motivado a asistir a clase", un 18,2 \% de los estudiantes están más bien de acuerdo, un 38,2 \% se mantiene neutral y un 5,5\% completamente en desacuerdo. Con respecto a la pregunta "El uso de Kahoot me ha motivado a permanecer más atento durante las clases” el 32,7\% de los estudiantes está más bien de acuerdo, el 25,5\% se mantiene neutral, un 1,8\% está más bien en desacuerdo y un 1,8\% está totalmente en desacuerdo.

En cuanto al aprendizaje percibido, 18 estudiantes (32,7\%) están completamente de acuerdo con la afirmación "Creo que Kahoot ha mejorado mi aprendizaje de la materia Derecho Romano”, 23 estudiantes (41,8\%) están más bien de acuerdo, 11 estudiantes se mantienen neutrales (20\%), 2 estudiantes (3,6\%) más bien en desacuerdo y 1 estudiante $(1,8 \%)$ completamente en desacuerdo. En resumen, un 74,5 \% de los estudiantes cree que ha mejorado su aprendizaje.

Como conclusiones de la gamificación con Kahoot, la mayoría de los estudiantes han encontrado la experiencia divertida y motivadora y consideran que ha mejorado su aprendizaje. No obstante, hay 3 estudiantes a los que no les ha gustado en absoluto esta experiencia (5\% de los estudiantes que han contestado la encuesta). 
A continuación se analizan las preguntas acerca de la Lección Moodle. La primera pregunta, "La Lección han cumplido el objetivo para el que estaba diseñada: repasar la asignatura" ha tenido un resultado absolutamente positivo, 45 estudiantes (81,8\%) están completamente de acuerdo y 10 estudiantes (18,2\%) están más bien de acuerdo.

En cuanto a la motivación, 36 estudiantes (65,5\%) están completamente de acuerdo con la afirmación "El formato de la Lección, en forma de juego, me ha motivado para realizarla”, 15 estudiantes (27,3\%) están más bien de acuerdo, 3 estudiantes (5,5\%) se muestran neutrales, y 1 estudiante (1,8\%) está complemente en desacuerdo.

\section{Preguntas abiertas sobre Kahoot}

Los efectos percibidos por los estudiantes en relación con el uso de Kahoot en las Magistrales se manifiestan en dos aspectos: respecto a la motivación y diversión, y respecto a los efectos sobre aprendizaje de la asignatura.

En primer lugar se muestra claramente la sorpresa y diversión que esta experiencia de gamificación ha causado. Los estudiantes consideran en general que ha sido una buena técnica que les ha motivado a prestar más atención en clase. Además, les ha permitido repasar jugando al final de la clase de forma amena, y ha roto la monotonía en la que puede caer la lección magistral. Todo esto ha provocado un buen ambiente en el grupo de estudiantes que se ha mantenido incluso hasta el día del examen final.

En cuanto al elemento de competición, a algunos estudiantes les ha generado un aliciente para mejorar el aprendizaje, les ha motivado a seguir estudiando para poder ganar y superarse con una "rivalidad sana" y un "ambiente desenfadado”. No obstante, algún estudiante ha apreciado que parte del colectivo se toma el juego más a broma que como actividad didáctica.

Los efectos importantes que sobre el aprendizaje perciben son los siguientes:

- Les ha ayudado a centrar los conocimientos y aclararlos, a aprender los conceptos fundamentales de cada Magistral y a comprobar los conocimientos adquiridos durante la clase y los conceptos que deben repasar.

- Lo consideran un entrenamiento satisfactorio para realizar exámenes tipo test y para pensar más rápido.

- En algunos casos les ha motivado a ir a clase con la lección aprendida y a llevar la asignatura día a día.

No obstante, algún estudiante considera que las pruebas Kahoot no tiene ningún efecto sobre el aprendizaje y que debería emplearse ese tiempo en otras cuestiones como la resolución de dudas.

A continuación se encuentran algunos comentarios de los estudiantes que muestran las percepciones mencionadas:

"Ha sido una experiencia divertida $\mathrm{Y}$ motivadora. Me parece muy acertado hacer el kahoot al final de la clase, por los siguientes motivos: supone una motivación extra a la hora de prestar atención a la clase, permite pasar los últimos minutos de clase, que es cuando más cansados estamos los alumnos, repasando de forma entretenida lo aprendido, ha promovido un ambiente desenfadado que nos ha hecho salir de clase con buen humor.”
"Mi experiencia ha sido totalmente satisfactoria, ha sido todo un descubrimiento realmente entretenido y divertido. Sin duda ameniza las clases y te ayuda en cierta medida a escuchar de forma más atenta para luego poder jugar bien y que se vean resultados”

"Desde luego ha sido una experiencia muy divertida y motivadora. Kahoot me ha ayudado a involucrarme más en la asignatura y superarme cada vez más intentando aprender y ganar el juego.”

"No solo rompe con la monotonía de una clase magistral sino que también motiva a ir a clase y además llevar la lección aprendida”

\section{Preguntas abiertas sobre la Lección moodle}

En general los estudiantes han percibido el juego del Templo de la Jurisprudencia como un facilitador de su aprendizaje. En concreto ha permitido repasar la asignatura de una forma amena y útil antes de enfrentarse al examen final. Algunos estudiantes también han manifestado que el juego les ha permitido conciliar estudio y diversión y han agradecido mucho las notas de humor que han encontrado a lo largo de las preguntas.

En cuanto a la longitud del juego y a pesar de la posibilidad de abandonar el juego para reanudarlo más tarde, algunos estudiantes piensan que es demasiado largo y que se puede hacer algo pesado debido al tiempo requerido para completarlo. En este sentido, un estudiante propone fraccionar el juego en fragmentos más cortos, otro argumenta que la longitud del juego es entendible dada la magnitud de la materia y un último estudiante afirma de forma muy entusiasta que “aunque sea muy largo, lo volvería a hacer mil veces”.

También existen comentarios de los estudiantes acerca de las preguntas incluidas en el juego, afirmando que las respuestas no estaban en sus apuntes o que no habían visto esa materia en clase. Estos estudiantes han ignorado el trabajo individual necesario para superar la asignatura y establecido en el plan de trabajo. Sin embargo, otros estudiantes consideran este hecho de forma positiva afirmando que el juego les ha permitido aprender “cosas nuevas”.

Los comentarios mencionados, y aparentemente contradictorios, acerca de la longitud del juego y del carácter de las preguntas confirman la existencia de dos tipos de actitudes en estos estudiantes: el interés por aprender o el interés por superar la asignatura con el menor esfuerzo posible.

A continuación se encuentran algunos comentarios de los estudiantes que muestran las percepciones mencionadas:

"Me ha encantado, como llevaba un hilo conductor "muy de la época" se ha hecho realmente amena. Además, ha ido tocando todos los temas que hemos visto y como repaso ayuda mucho. Una asignatura cambia mucho cuando es algo más que tragar libros y, a pesar de que la materia no es especialmente ligera, ha ayudado a que se lleve de manera más asequible.”

"Este juego ha estado muy bien, lo he encontrado divertido aunque tal vez un poco pesado ya que, había muchas preguntas. Sin lugar a dudas ha sido un gran juego para repasar la asignatura antes de presentarme al examen. Estoy muy contenta con este método de estudio y creo que es altamente recomendable para fomentar y conciliar el estudio y la diversión.” 
“Juego muy divertido además el humor en las preguntas lo hace muchísimo más ameno. Realmente lo mejor de la asignatura, se ve que ha sido un trabajo largo realizarlo pero la experiencia es muy buena. Mi grupo de amigos y yo estamos muy contentos y nos lo hemos pasado muy bien jugando.”

\section{Resultados generales}

Si se realiza una triangulación de los resultados obtenidos a través de los distintos instrumentos de recogida de datos aparecen tres efectos principales tras las experiencias de gamificación diseñadas: diversión, motivación y aprendizaje.

\section{Diversión}

La diversión ha sido el efecto principal que las experiencias de gamificación han causado en los estudiantes. Los resultados del estudio sugieren que la diversión puede propiciar un ambiente en clase distendido y motivador, pero también puede llevar a que las pruebas de evaluación no se consideren como algo serio, e incluso, en algún caso, a que no se produzca ningún aprendizaje. Por otra parte, se conoce que el aprendizaje provoca diversión (Villagrá-Arnedo, Gallego-Durán, MolinaCarmona \& Llorens-Largo, 2016). En ediciones posteriores de esta experiencia sería necesario profundizar en la relación entre diversión, motivación y aprendizaje.

\section{Motivación}

Las experiencias de gamificación han motivado a muchos estudiantes a asistir a clase, a permanecer más atentos y a la realización de las pruebas de evaluación. Por otra parte, la mayoría de los estudiantes han manifestado que se han visto fuertemente motivados por la competición para ganar el juego, y otros no parecen motivados ante de la idea de aprender mediante un juego. En general, los resultados del cuestionario han mostrado que las experiencias de gamificación han provocado distintos niveles de motivación en los estudiantes, debido a sus diferencias individuales. Sería necesario profundizar en estas diferencias para comprender los efectos de la gamificación en cada uno de los estudiantes.

\section{Aprendizaje}

La mayoría de los estudiantes han percibido las experiencias de gamificación como facilitadoras del aprendizaje y piensan que han mejorado el aprendizaje de la materia Derecho Romano gracias a ellas. Es necesario tener en cuenta que en este análisis preliminar se ha tenido en cuenta únicamente el aprendizaje percibido por los estudiantes. En la siguiente etapa de análisis se analizarán las pruebas de evaluación formativa y se estudiará la evolución del aprendizaje de cada uno de los estudiantes.

\section{CONCLUSIONES}

Este trabajo ha presentado un estudio empírico acerca de los efectos sobre el aprendizaje provocados por dos experiencias de gamificación en la asignatura Derecho Romano. La descripción detallada de las experiencias permite que puedan ser tomadas como referencia por otros profesores de Derecho Romano, de las Ciencias Jurídicas e incluso de otras disciplinas afines en cuanto a evaluación se refiere.

De acuerdo con los resultados obtenidos y con las diferencias individuales entre los estudiantes, se recomienda que las experiencias de gamificación sean voluntarias, estén circunscritas a determinadas actividades didácticas y tengan algún tipo de recompensa en la nota final de la asignatura.

\section{REFERENCIAS}

Adesope, O. O., Trevisan, D. A. \& Sundararajan, N. (2017). Rethinking the Use of Tests: A Meta-Analysis of Practice Testing. Review of Educational Research, 87(3), 659-701.

Bloom, B. S., Madaus, G. F. \& Hastings, J. T. (1981). Evaluation to improve learning. McGraw-Hill.

Bodnar, C. A., Anastasio, D., Enszer, J. A. \& Burkey, D. D. (2016). Engineers at Play: Games as Teaching Tools for Undergraduate Engineering Students. Journal of Engineering Education, 105(1), 147-200.

Bouki, V., Economou, D. \& Kathrani, P. (2014). Gamification and legal education: A game based application for teaching university law students. In 2014 International Conference on Interactive Mobile Communication Technologies and Learning (IMCL2014) (pp. 213-216). IEEE.

Deterding, S. (2015). The Lens of Intrinsic Skill Atoms: A Method for Gameful Design. Human-Computer Interaction, 30(3-4), 294-335.

Dicheva, D., Dichev, C., Agre, G. \& Angelova, G. (2015). Gamification in Education: A Systematic Mapping Study. In Interactive Mobile Communications Technologies and Learning (Vol. 18, pp. 75-88).

Salen, K. \& Zimmerman, E. (2003). Rules of play: Game design fundamentals. Cambridge, MA: MIT Press.

Stake, R. E. (1978). The Case Study Method in Social Inquiry 1. Educational Researcher, 7(2), 5-8.

Villagrá-Arnedo, C., Gallego-Durán, F. J., Molina-Carmona, R. \& Llorens-Largo, F. (2016). PLMan: Towards a Gamified Learning System. In P. Zaphiris \& A. Ioannou (Eds.), Learning and Collaboration Technologies: Third International Conference, LCT 2016, Held as Part of HCI International 2016, Toronto, ON, Canada, July 1722, 2016, Proceedings (pp. 82-93). Cham: Springer International Publishing.

Wittrock, M. C. (1989). La investigación de la enseñanza, I. Enfoques, teorías y métodos. Madrid: Ediciones PAIDOS.

\section{ANEXO}

Kahoot: 1. Ha sido divertido competir con otros, 2. Ha sido divertido jugar todos juntos en el aula, 3 . El uso de Kahoot me ha motivado a asistir a clase, 4. El uso de Kahoot me ha motivado a permanecer más atento durante las clases, 5. Creo que Kahoot ha mejorado mi aprendizaje de la materia Derecho Romano, 6. ¿Cómo describirías tu experiencia con Kahoot? ¿Te ha parecido divertida, aburrida, motivadora, desmotivadora? ¿Por qué?, 8. ¿De qué forma te ha ayudado Kahoot a mejorar el aprendizaje de la asignatura Derecho Romano? Lección: 1. La Lección ha cumplido el objetivo para el que estaba diseñada: repasar la asignatura 2. El formato de la Lección, en forma de juego, me ha motivado para realizarla, 3 ¿Qué mejorarías? ¿Qué es lo que más te ha gustado? ¿Qué otros comentarios realizarías? 